\title{
(a draft of) Non-secure Client Awareness in TF-M
}

This paper was downloaded from TechRxiv (https://www.techrxiv.org).

\section{LICENSE}

CC BY 4.0

SUBMISSION DATE / POSTED DATE

06-01-2021 / 07-01-2021

CITATION

Iannillo, Antonio Ken; Rivera, Sean; Suciu, Darius; Sion, Radu; State, Radu (2021): (a draft of) Non-secure Client Awareness in TF-M. TechRxiv. Preprint. https://doi.org/10.36227/techrxiv.13525547.v1

DOI 


\section{(a draft of) Non-secure Client Awareness in TF-M}

v0.1

\author{
Antonio Ken Iannillo \\ SnT, University of Luxembourg \\ antonioken.iannillo@uni.lu \\ Radu State \\ SnT, University of Luxembourg \\ radu.state@uni.lu
}

\author{
Sean Rivera \\ SnT, University of Luxembourg \\ sean.rivera@uni.lu
}

\author{
Darius Suciu \\ Stony Brook University \\ dsuciu@cs.stonybrook.edu
}

\begin{abstract}
Radu Sion
Stony Brook University

sion@cs.stonybrook.edu
\end{abstract}

\begin{abstract}
Ken says:add shorter version of the intro ( 10 lines) The main contribution of this paper consists of a novel client identification and authentication approach completely handled in the TEE, reducing its attack surface. We implemented our solution for armv8-m architecture supporting Trustzone technology, showing no need to change the source code of the Rich OS. Empirical results showed its effectiveness, while the size of TCB increased only by XX\%.
\end{abstract}

\section{Introduction}

Software vulnerabilities can closely affect our daily lives since IoT devices might monitor our health, drives our cars, control our homes, or assist our factories. As these embedded systems are getting more connected and widely adopted, trusted execution environments are emerging as a cybersecurity solution to malicious attacks.

A Trusted Execution Environment (TEE) is an execution environment that protects both its runtime states and stored assets. Since operating systems are usually large and complex (aka Rich OS), they are more prone to the introduction or the regression of software bugs. Still, a TEE protects its data even if the OS is compromised. Typical TEE use cases are the secure storage and usage of cryptographic keys, the protection of digital copyrighted information, or the safe processing of biometric sensor data. ARM TrustZone technologies aided smartphones to protect security-critical assets from potential breaches in the broader set of functionalities. ARM recently introduced TrustZone for microprocessors, namely TrustZone-M (TZ-M) ${ }^{1}$, intending to protect also IoT devices that are becoming ubiquitous and potentially more dangerous than smartphones if vulnerable to attacks.

Enforced directly by hardware extensions in the processor, TZ-M-enabled embedded systems consist of a normal (non-secure) world (NW) restricted with few resources and a secure world (SW) with no restrictions. They are fully isolated where generic functionalities are in the NW while the SW implements a Trusted Execution Environment

1. https://developer.arm.com/ip-products/security-ip/trustzone/ trustzone-for-cortex-m
(TEE). These two worlds need to communicate, but they are unaware of the logic implemented in the other world. In particular, the secure world does not know which task with which capabilities requests a secure service. An attacker can exploit this semantic gap and fool the SW into misusing its unrestricted capabilities: a non-authorized task can break the NW's security mechanisms by making a simple request to the SW, unaware to whom it's granting its services.

This paper presents SOLUTION_NAME, a novel mechanism implemented in the SW to identify and authorize secure service call from the NW. While the current solution needs changes of the non-secure software (typically an RTOS), SOLUTION_NAME exploits available hardware (i.e., the memory protection unit or MPU) to handle clients identification and authorization in a transparent way to non-secure software. Further, our solution binds the identification mechanism to the secure service call instead of the non-secure context switch as it happens in $\mathrm{tf}-\mathrm{m}$. We implemented SOLUTION_NAME on top of the reference implementation of a TZ-m-enabled secure firmware, namely trustedfirmware-m (tf-m $)^{2}$, and we demonstrated its effectiveness.

Formally, we investigate the following research questions:

RQ1 Does SOLUTION_NAME provides TZ-M-enabled embedded systems an effective authentication mechanism?
RQ1.1 How an attacker can impair SOLU- TION_NAME and impersonate another client?

RQ2 What's the impact of SOLUTION_NAME on the code?

RQ2.1 What's the size of code changes in the nonsecure software?

RQ2.2 What's the size of the secure software, i.e., the trusted computing base (TCB)?

RQ3 What's the overhead of SOLUTION_NAME on the performance?

\footnotetext{
2. https://www.trustedfirmware.org/projects/tf-m/
} 


\section{Background}

security states non-secure, non-secure callable, and secure states. transitions between states

non-secure memory protection in NW NW is a RTOS. User mode. MPU, MPU regions, and access control. secure mechanisms to preserve non-secure memory from unauthorized access.

security services What kind of service should be secured in the SW? Simple and fast. Crypto, Storage, and Remote Attestation APIs. Use of identification in secure services.

\section{Threat Model}

We consider the trust boundary between the SW and the NW, established through the TZ-M technology implemented in embedded devices. Data from the NW should not be trusted by the SW that must validate them in order to ensure the confidentiality and integrity of the secure data. In particular, we consider those secure data stored in the SW by a non-secure application, while non-secure applications are isolated to each other in the NW by the non secure OS.

When a non-secure application makes a request to the SW, it's presented with an id that is used to determine the rights to access a specific service or datum. In order to preserve isolation, different ids are assigned to different entities in the NW. Thus, each non-secure application can be treated differently by the SW with regard to access to services and data.

A malicious application may make a request to the secure world pretending to be another non-secure application, presenting the target's id instead of its own.

\section{Existing Solutions}

solution in trustzone-A In systems powered by cortex A processors, trustzone extensions work differently from TZM. Security state changes pass through the monitor. The non secure OS attaches the process id (pid) of the caller application to each request to the secure world.

via code hash [1] via digital signature [2]

context management apis what they are? how they work? reference to global platform TEE api implementation in trustedfirmware-m

\section{SOLUTION_NAME}

Existing solutions assign client ids in non secure privileged code since the non secure OS is also in charge of guaranteeing application isolation in the NW. SOLUTION_NAME also depends on the non-secure OS to isolate correctly the non-secure applications but it does not require any change in the non secure OS's code or any additional secure API available to the NW.

The non secure OS isolates non-secure applications in threads. Each thread is isolated from each other and from the kernel. Isolation prevents access to memory, device drivers, or kernel objects not specifically granted. Memory protection is implemented through Memory Protection Unit (MPU) hardware. Any application thread will need access to its own stack buffer. On context switch, a dedicated nonsecure MPU region will be programmed with the bounds of the stack buffer. Each of these MPU regions are unique since there are no two threads that share the same stack. Thus, they can be used to uniquely identify a non-secure application.

When a non-secure application requests a secure service, the non-secure MPU is configured with the aforementioned region. The request passes through a veneer and handled by the secure partition manager (SPM) that dispatches it to the secure service. SOLUTION_NAME is implemented in the SPM and, upon reception of a request from the $\mathrm{NW}$, provides the client id by reading the non-secure MPU configuration. Algorithm 1 presents the pseudocode of $S O$ LUTION_NAME's implementation.

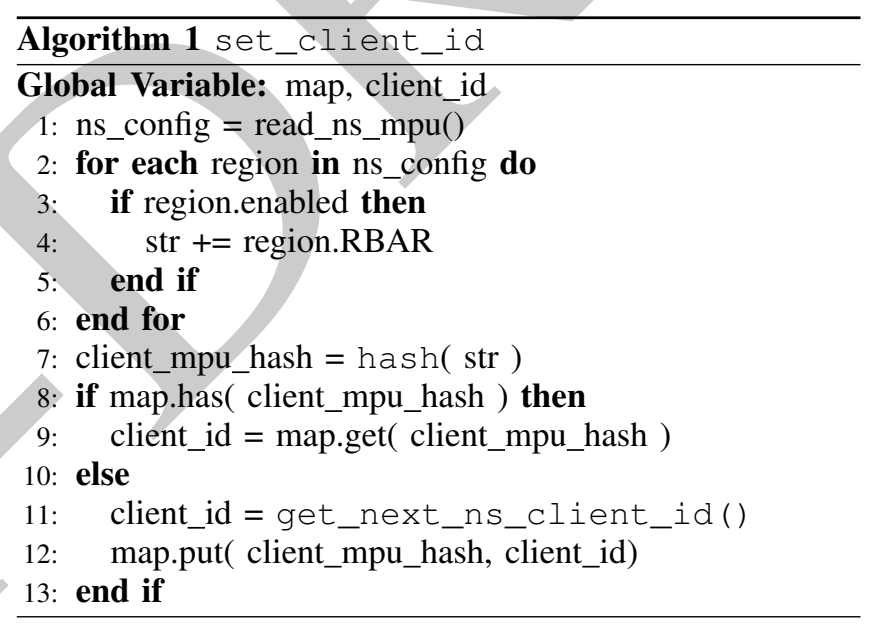

We implemented SOLUTION_NAME in $\mathrm{tf}-\mathrm{m}$. In the IPC model, set_client_id is called from the SVC_Handler_IPC function ${ }^{3}$. In the library model, it is called from the tfm_core_partition_request function $^{4}$. In both cases, it's defined as a function in $t \mathrm{fm}$ nspm.h. Once we read the non secure MPU configuration, we concatenate the base addresses of the enabled regions (lines 2-6). This byte array is unique to the client since it contains at least a read/write region for its stack that no other clients can access in the NW. We use the hash of such array to retrieve the client id assigned to it. If none is assigned, we assign a new id using the get_next_ns_client_id function (line 11).

further consideration on id uniqueness The client id is unique to the thread, because we consider all the MPU regions that include the $\mathrm{R} / \mathrm{W}$ region of the thread stack. An optimization in line 3 is to consider only $\mathrm{R} / \mathrm{W}$ regions.

Some system can isolate also the code of the different application, that can have in turn different threads. It may

3. secure_fw/spm/cmsis_psa/tfm_core_svcalls_ipc.c

4. secure_fw/spm/cmsis_func/tfm_secure_api.c 
be necessary to uniquely identify the application and not each of its threads. To implement code isolation, on context switch, another dedicated non-secure MPU region will be programmed with the bounds of the code area. This region is read only and it will be unique to the application and its threads. Thus, an implementation would be to consider only $\mathrm{R} / \mathrm{O}$ regions in line 3 .

Similarly, there can be systems where there is a need for a thread client id and an application/group client id. In this case, both of the previous cases can be implemented side by side.

\section{Verification and Discussion}

test suite of trusted firmware-m specific functional tests

what happen is ctx mgmt apis are hooked? what if non-secure OS is compromised? what happen if hidden pointers are used?

\section{Conclusion}

\section{Acknowledgments}

The authors would like to thank...

\section{References}

[1] H. Jiang, R. Chang, L. Ren, W. Dong, L. Jiang, and S. Yang, "An effective authentication for client application using arm trustzone," in International Conference on Information Security Practice and Experience, pp. 802-813, Springer, 2017.

[2] L. Catuogno and C. Galdi, "A fine-grained general purpose secure storage facility for trusted execution environment.," in ICISSP, pp. 588$595,2019$. 